PAPER

\title{
Effects of lesions of the human posterior thalamus on ocular fixation during voluntary and visually triggered saccades
}

\author{
R Rafal, M McGrath, L Machado, J Hindle
}

See end of article for authors' affiliations

J Neurol Neurosurg Psychiatry 2004;75:1602-1606. doi: 10.1136/jnnp.2003.017038

..................

Correspondence to: B Rafal, School of Psychology, University of Wales, Bangor, Brigantia Building, Penrallt Road, Bangor, Gwynedd LL57 2AS, UK;

r.rafal@bangor.ac.uk

Received 16 April 2003 In revised form

29 December 2003

Accepted 5 February 2004
Objective: To investigate the role of the posterior thalamus in controlling voluntary and visually triggered eye movements and ocular fixation.

Methods: The latency to initiate saccades to peripheral targets (visually triggered) and in response to verbal commands (voluntary) was measured in three patients with unilateral lesions of the posterior thalamus, in normal controls, and in neurological controls with Parkinson's disease. On half the trials a fixation point offset simultaneously with target onset, and on half it remained visible.

Results: Offset of the fixation point simultaneous with target onset decreased saccade latency for both voluntary and visually triggered eye movements in controls, but only for voluntary saccades in patients with thalamic lesions.

Conclusions: These findings suggest that separate neural systems control fixation when making voluntary and visually triggered eye movements, and that the thalamus is involved in the control of fixation for visually triggered but not for voluntary saccades.
$\mathrm{T}$ he thalamus is a nexus connecting the subcortical and cortical oculomotor centres that orchestrate the coordination of voluntary and reflexive eye movements necessary for coherent visually guided behaviour. An oculomotor function for the central and posterolateral thalamus has been suggested by animal studies showing that saccades can be elicited by electrical stimulation of thalamic nuclei ${ }^{1-3}$ and that single units in them are active in relation to saccades..$^{5}$ Abnormalities of voluntary and visually triggered saccades have been reported in patients with acute thalamic lesions, ${ }^{6-9}$ most of whom had hemispatial neglect. It is not clear from these reports whether the thalamus has a specific oculomotor function, or whether the deficits reported reflect the role of the thalamus in the control of visual attention. The current study examined the effect of chronic lesions of the posterior thalamus on voluntary and visually triggered saccades in three patients (none of whom had signs of neglect). It also examined the role of the thalamus in the control of ocular fixation by employing a fixation offset manipulation. On half the trials a fixation point offset at the time a target was presented instructing the saccade, while on the other half of the trials the fixation point remained visible until the saccade was initiated. In normal individuals, the presence of a fixation point temporally overlapping presentation of the target increases the latency for both visually triggered and voluntary saccades. ${ }^{10}{ }^{11}$

\section{METHODS}

\section{Participants}

Three patients with thalamic lesions who had sustained haemorrhages centred in the posterior thalamus (fig l) were tested 10-16 months later after obtaining informed consent. One patient was a woman with a right sided lesion (age 54) and two were men with left sided lesions (ages 54 and 61). All were independent with varying degrees of contralesional motor and sensory deficits. None had any obvious oculomotor deficits at the time of testing, and none had any signs of visual field defect, hemispatial neglect, or visual extinction on conventional bedside tests: confrontation testing of visual fields and extinction; drawing and cancellations tasks. In addition, 16 neurologically normal individuals (nine men and seven women, mean age 67.5 years) and 14 hypokinetic patients with idiopathic Parkinson's disease (nine men, five women, mean age 66 years; all on medications) were also tested. We previously examined the effect of chronic lesions of frontal and parietal cortices on ocular fixation, ${ }^{12}$ and this study included a group of patients with subcortical pathology, not involving the thalamus, as a further neurological control.

\section{Procedure}

Saccade latency was measured with an Eye Trac 210 scleral reflectance device (Applied Science Laboratories, Bedford, MA) interfaced with a PC computer. "Presentation" software was used to present stimuli and to sample eye position at $1000 \mathrm{~Hz}$. This output was analysed with custom software to calculate saccade latency and direction when threshold velocity reached $50 \mathrm{deg} / \mathrm{sec}$.

After an inter-trial interval of $2500 \mathrm{~ms}$, each trial began with a display (presented on a NEC Multisync video graphics array monitor; Mitsubishi, Japan) consisting of a black background on which were a white fixation point and two unfilled $2.4^{\circ}$ white squares located $10^{\circ}$ to the right and left. Participants maintained their gaze on the fixation point (a white filled circle measuring $0.7^{\circ}$ ) for a variable interval of time ranging between $700 \mathrm{~ms}$ and $1300 \mathrm{~ms}$, after which a target was presented instructing them to make an eye movement towards one of the peripheral boxes. In separate blocks, saccades were made either to visual stimuli (a white, $1.8^{\circ}$ asterisk that appeared, randomly, in the left or right box and remained visible until response) or in response to the words "right" or "left" (presented randomly from a speaker in front of the participant). For both tasks, on a random half of the trials the fixation point offset simultaneously with target presentation and, on the other random half of the trials, the fixation point remained visible.

The experimenter monitored eye position on a slave scope that replicated the display viewed by the participants and also plotted a cursor indicating eye position recorded by the Eye

Abbreviations: $\mathrm{FOE}$, fixation offset effect 


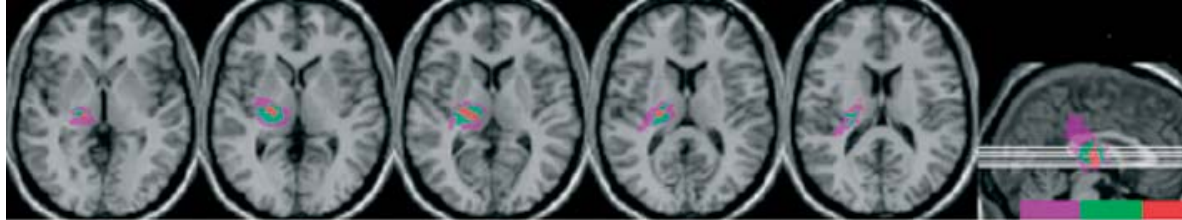

Patient 1

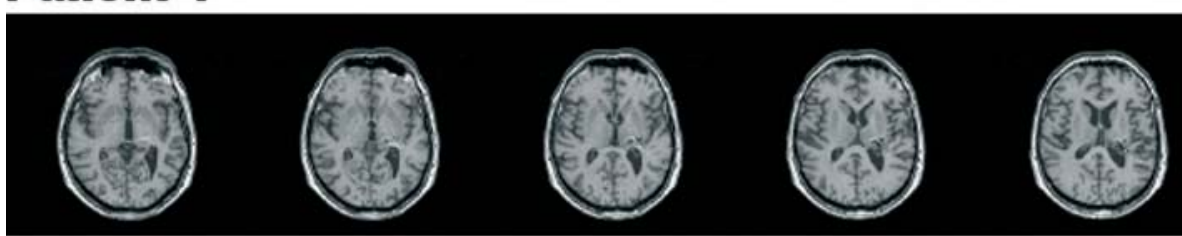

Patient 2

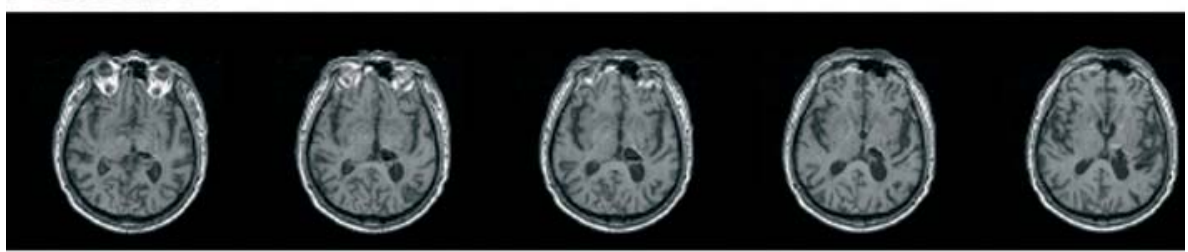

Patient 3

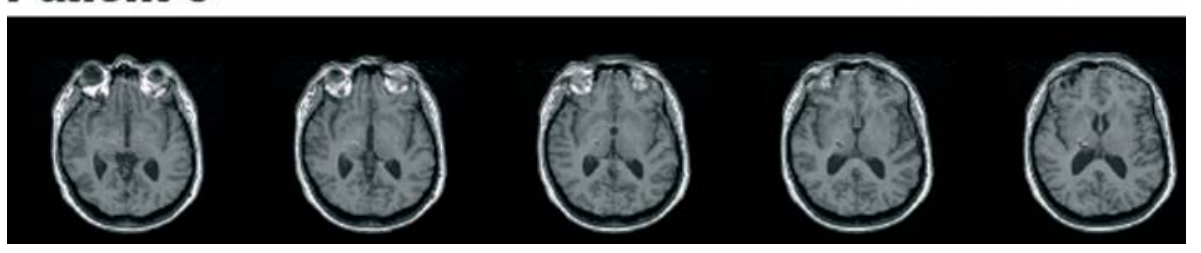

Figure $1 \quad$ Tl-weighted magnetic resonance imaging scans of the three patients with lesions centred in the posterior thalamus, obtained with $1 \mathrm{~mm}$ slices. The top panel depicts the regions of overlap. Lesions were reconstructed (http://www.cla.sc.edu/ psyc/faculty/rorden/mricro.html) and normalised using statistical parametric mapping. The colour scale indicates the number of patients with a lesion involving that area: purple, one patient; green, two patients; red, all three patients.
Trac. The trials on which the participants failed to maintain fixation, or blinked, before the target was presented were marked as "bad" trials by the experimenter by pressing the mouse and were excluded from subsequent analysis.

Experimental sessions consisted of one block of the visual target task and one block of the auditory target task, with 80 trials in each block. Two of the patients with thalamic lesions were tested in five sessions and one was tested in six sessions, with the order of the tasks counterbalanced across testing sessions. Each control was tested in one block of each task in a single session with the order counterbalanced across successive subjects.

\section{RESULTS}

\section{Errors}

For the control groups, the trials excluded from the analysis due to a failure to maintain fixation or blinking were infrequent: $3 \%$ of trials were excluded for the normal controls and 1\% for the patients with Parkinson's disease. In contrast, $15 \%$ of trials were excluded for the patients with thalamic lesions. With visual targets, errors in which a saccade was made in the wrong direction were rare $(<2 \%$ for all groups). The three groups did not differ in the number of direction errors when making voluntary saccades (normal 9\%, Parkinson's 8\%, thalamic lesion 7\%).

\section{Saccade latencies}

Normal controls

After excluding responses greater than three standard deviations from the mean, the mean saccade latency for correct responses was calculated for each condition in each subject. A $2 \times 2 \times 2$ repeated measures ANOVA included, as within subject factors, task (voluntary saccade $v$ visually triggered saccade), field (left $v$ right saccade), and fixation (offset $v$ overlap). Saccades had shorter latencies to visual targets than in response to verbal commands $\left(F_{1,15}=85.9\right.$, $\mathrm{p}<0.001$ ), and when the fixation point offset simultaneously with the target presentation, than when the fixation point overlapped the target $\left(F_{1,15}=14.6, \mathrm{p}=0.002\right)$.

Controls with Parkinson's disease

After excluding responses greater than three standard deviations from the mean, mean saccade latency for correct responses was calculated for each condition in each patient, and an ANOVA was conducted as for the normal participants. As shown in fig 2, the results of the patients with Parkinson's disease were similar to those of the normal controls. The

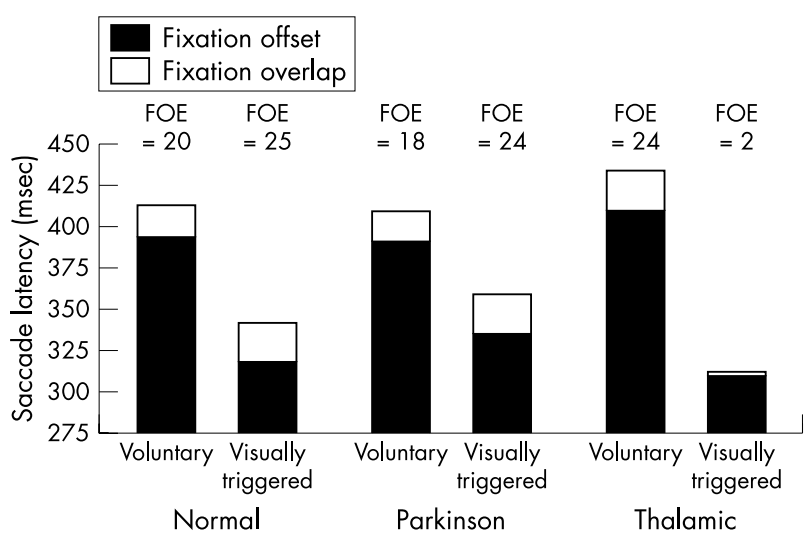

Figure 2 Mean latencies (in msec) of the normal controls, patients with Parkinson's disease, and patients with thalamic lesions for voluntary saccades (auditory target) and visually triggered saccades (visual target). The white areas show the benefit of fixation point offset. FOE, fixation offset effect. 
Table 1 Mean saccade latency (in $\mathrm{msec}$ ) for the three patients with thalamic lesions in each condition

\begin{tabular}{|c|c|c|c|c|c|c|c|c|}
\hline & \multicolumn{4}{|c|}{ Voluntary saccade } & \multicolumn{4}{|c|}{ Visually triggered saccade } \\
\hline & \multicolumn{2}{|c|}{ Contralesional } & \multicolumn{2}{|l|}{ Ipsilesional } & \multicolumn{2}{|c|}{ Contralesional } & \multicolumn{2}{|l|}{ Ipsilesional } \\
\hline & Offset & Overlap & Offset & Overlap & Offset & Overlap & Offset & Overlap \\
\hline Patient 1 & 436 & 453 & 412 & 429 & 381 & 375 & 347 & 342 \\
\hline Patient 2 & 492 & 489 & 408 & 438 & 312 & 314 & 269 & 271 \\
\hline Patient 3 & 372 & 412 & 342 & 385 & 276 & 286 & 276 & 285 \\
\hline Mean (SEM) & $433(12.0)$ & $451(10.7)$ & $387(11.7)$ & $417(9.7)$ & $323(6.8)$ & 325 (9.3) & $297(6.5)$ & $299(7.1)$ \\
\hline
\end{tabular}

saccades had shorter latencies to visual targets than in response to verbal commands $\left(F_{1,13}=21.9, \mathrm{p}<0.001\right)$ and when the fixation point offset compared with when the fixation point overlapped the target $\left(F_{1,13}=17.2, \mathrm{p}=0.001\right)$.

\section{Patients with thalamic lesions}

For each session, after excluding responses greater than three standard deviations from the mean, mean saccade latency for correct responses was calculated for each condition in each patient. A $3 \times 2 \times 2 \times 2$ mixed ANOVA, with session as the random factor, included "patient" as a between subject variable and, as within subject factors, task (voluntary saccade $v$ visually triggered saccade), field (contralesional $v$ ipsilesional saccade), and fixation (offset $v$ overlap). The results are given in tables 1 and 2 and in fig 2. Saccade latencies were longer for voluntary saccades than for visually triggered saccades $\left(F_{1,13}=124.5, \mathrm{p}<0.001\right)$. Saccade latencies were shorter when the fixation point offset for voluntary saccades, but not for saccades to visual targets $\left(F_{1,13}=11.3\right.$, $\mathrm{p}=0.005)$. All three patients showed a difference in the fixation offset effect (FOE) for the two tasks (table 2), and the difference in FOE magnitude did not differ among the three patients $\left(F_{1,2}=0.44\right)$. Separate ANOVAs for each saccade task confirmed that the FOE was statistically reliable in the voluntary saccade task $\left(F_{1,15}=17.3, \mathrm{p}=0.001\right)$, whereas there was no effect of fixation offset for the visually triggered saccade task $\left(F_{1,15}=0.14\right)$.

The $95 \%$ confidence interval of the difference for the FOE magnitude in the visually triggered saccade task for the normal subjects was $6.7 \mathrm{msec}$ to $32.4 \mathrm{msec}$. As shown in table 2, in two of the three patients with thalamic lesions the magnitude of the FOE was below the lower limit of this range, and in the third patient (patient 3 ) it was at the lower limit of this range ( $9 \mathrm{msec}$ ); in this patient, the FOE effect in this task did not approach statistical reliability $\left(F_{1,5}=3.1\right.$, $\mathrm{p}=0.14$ ).

Critically, not only did the patients with thalamic lesions not show any FOE for the visually triggered saccade task, but, as the ANOVA reported above revealed, in contrast with both control groups, they also showed a reliably smaller FOE in the visually triggered saccade task than in the voluntary saccade task. In normal participants, the $95 \%$ confidence interval of the difference for the FOE magnitude for the two tasks- that is, voluntary saccade task FOE minus visually triggered saccade task FOE, was $-19.4 \mathrm{msec}$ to $9.4 \mathrm{msec}$. All three patients fell outside this range: patient $1=23 \mathrm{msec}$, patient $2=12 \mathrm{msec}$, and patient $3=33 \mathrm{msec}$.

A marginally significant three way interaction of patient $\times$ field $\left(F_{1,13}=3.04, \mathrm{p}=0.083\right.$ reflected the fact that one patient (patient 2) had longer latencies for contralesional saccades than for ipsilesional saccades for both the voluntary saccade task $\left(F_{1,4}=13.0, \mathrm{p}<0.025\right)$ and for the visually triggered saccade task $\left(F_{1,4}=15.3, \mathrm{p}=0.017\right)$. The effect of field did not approach statistical reliability for either task in patients 1 and 3 ( $p>0.13$ in all cases).

\section{DISCUSSION}

The novel finding of the current study is that chronic lesions of the posterior thalamus have a specific oculomotor effect on the neural control of ocular fixation. A fixation offset manipulation was employed to measure the degree to which a visual stimulus at fixation inhibited the initiation of either voluntary or visually triggered saccades. The patients with thalamic lesions showed normal FOE for voluntary saccades, but no FOE for visually triggered saccades. Moreover, neither the Parkinson's controls reported here, nor patients with parietal or frontal damage whom we recently studied, ${ }^{12}$ failed to show any inhibitory effect of a fixation point on visually triggered saccades.

Although the region of common lesion overlap in these three patients is the posterior thalamus, it is also the case that, in addition to damage to thalamic nuclei, there is also disruption of fibres of passage-in particular, the posterior limb of the internal capsule. Consideration must be given to the possibility that the abnormality of ocular fixation could be due to interruption of the fibres connecting the parietal cortex with the superior colliculus. However, since the same findings were not observed in patients with parietal lesions, ${ }^{12}$ this account seems less likely.

The cells controlling the visual grasp reflex towards new signals appearing in the visual periphery are located in the superior colliculi. ${ }^{13}{ }^{14}$ In the rostral pole of each colliculus are cells that are active during fixation (even in the dark) and whose activity is further increased by a visual signal at fixation. These fixation, or pole, neurones help keep the eyes from moving. Caudal to the fixation neurones, and inhibited by them, are cells (commonly referred to as movement cells) whose activity helps the eyes move to a new position. Visually triggered eye movements, then, are controlled by an opponent process involving intrinsic collicular circuits: there is mutual inhibition between the visual grasp reflex, activated by abrupt signals in the visual periphery and mediated by more caudal movement cells, and the fixation reflex, activated by visual signals at fixation and mediated by rostral pole neurones. Together, the activity of these two types of cell determines when and where the eyes will move. ${ }^{15}$

\begin{tabular}{|c|c|c|}
\hline & $\begin{array}{l}\text { Voluntary } \\
\text { saccade }\end{array}$ & $\begin{array}{l}\text { Visually triggered } \\
\text { saccade }\end{array}$ \\
\hline Patient 1 & 17 & -6 \\
\hline Patient 2 & 14 & 2 \\
\hline Patient 3 & 42 & 9 \\
\hline Mean & 24 & 1.6 \\
\hline
\end{tabular}


The offset of a fixated stimulus prior to, or simultaneously with, the onset of a peripheral target disinhibits the visual grasp reflex and speeds reaction time to initiate an eye movement to the target. The benefit of fixation offset on saccadic latencies was first reported by Saslow ${ }^{16}$ and has been termed the fixation offset effect (FOE) ${ }^{17}$ Fixation offset paradigms compare latencies to make eye movements when a fixation stimulus remains present at target onset with those when the fixation stimulus offsets. The FOE is thought to result from changes in cellular activity in local inhibitory circuits within the superior colliculus that are triggered by the offset of a fixated stimulus. ${ }^{18}$ When a fixated stimulus offsets, the activity of fixation neurones decreases and eye movement latency is reduced. ${ }^{14}{ }^{19}$ Conversely, stimulating fixation neurones just prior to or during an eye movement can delay or arrest the eye movement. ${ }^{18}$ This finding is consistent with the demonstration that unilateral microstimulation of the rostral superior colliculus inhibits movement cells in both colliculi. ${ }^{13}$ 18 Together, these neurophysiological findings suggest that when fixation cell activity decreases in response to fixation offset, movement cell activity increases, disinhibiting the visual grasp reflex and speeding reaction time to initiate an eye movement to a peripheral target. In normal individuals the FOE is present for voluntary saccades as well as for saccades to visual targets. ${ }^{10}$ We have recently shown that normal individuals can strategically modulate the FOE independently for these two types of saccadic eye movements, ${ }^{11}$ and have suggested that the control of fixation for these two types of eye movement might have different neural substrates.

In the current study we confirmed that normal older individuals, as well as patients with Parkinson's disease, have an FOE for both voluntary and visually triggered eye movements. In patients with thalamic lesions, a normal FOE was observed for voluntary saccades but no FOE for visually triggered saccades. This dissociation provides further evidence that the control of fixation for these two types of eye movements have different neural substrates. These results suggest that the thalamus is part of the neural circuitry controlling fixation for visually triggered saccades but not for voluntary saccades.

Bender and Baizer ${ }^{20}$ have previously attempted to examine the effect of kainic acid lesions of the thalamus on ocular fixation in monkeys. They employed a "step paradigm", similar to that used to study visually triggered saccades in experiment 1 , except that offset and overlap conditions were tested in separate blocks. They observed no effect of kainic acid lesions on saccade latencies for either offset or overlap conditions (although, interestingly, their figures do reveal a trend for saccade latencies to be shorter after lesioning, similar to that reported here). They concluded that the thalamus does not play a role in controlling eye movements, and suggested that the effects of thalamic lesions on eye movements reported in earlier studies of humans and animals might have been due to damage to fibres of passage. However, in the pre-lesion testing, their paradigm did not produce an FOE- that is, saccade latencies were not shorter in the "step" condition than in the overlap condition even in normal monkeys. Their study, therefore, was unable to test for an effect of thalamic inactivation on the inhibitory influence of a fixation stimulus.

The absence of the FOE for visually triggered saccades in patients with thalamic lesions could reflect either a failure of fixation neurones to be activated by the fixation point, or a failure of fixation neurones to be released from inhibition when the fixation point offset. Since latencies of visually triggered saccades in patients, for both offset and overlap conditions, were comparable to the latencies of controls in the offset condition (see fig 2), it seems likely that thalamic lesions reduce the responsiveness of collicular fixation neurones to visual stimuli in their receptive fields. This loss of the inhibitory influence of a signal at fixation results in a disinhibition of visually triggered saccades to peripheral visual signals.

It is worth noting that, not only was the FOE preserved for voluntary saccades, but that its magnitude was comparable to controls. In a previous study of a patient with surgical resection of the pulvinar it was reported that, in a visual search task (that is, a task involving voluntary eye movement), there was an abnormal prolongation of fixation on each stimulus. ${ }^{8}$ We found no evidence for this kind of "sticky fixation" during voluntary eye movements in our patients with chronic lesions.

The inclusion of Parkinson's disease patients as neurological controls for the current study was motivated by two recent studies by Briand and colleagues in which visually guided saccade latencies were compared with those in normal controls under conditions of fixation offset ${ }^{21}$ and fixation overlap. ${ }^{22}$ Fixation offset was not manipulated within the same experiment but, rather, in two separate studies. Parkinson's disease patients had shorter saccade latencies in the experiment where fixation overlapped the saccade target, but were not different from controls in the "gap" condition in which fixation offset. Together these results raised the possibility that, like the patients with thalamic lesions reported here, the presence of a fixation point might have less of an inhibitory effect on visually triggered saccades in Parkinson's disease. However, the current study, in which fixation offset was systematically manipulated within blocks within the same experiment, revealed normal latencies, compared with normal controls, for both offset and overlap conditions. Moreover, we report the novel finding that the effect of a fixation point on voluntary saccades is also not different from normal controls.

One of the three patients (patient 2) had longer latencies for contralesional than for ipsilesional saccades for both voluntary and visually triggered saccades. Figure 1 reveals, however, that the lesion in this patient not only resulted in more complete destruction of the pulvinar than in the other two patients but that, unlike the other two patients, his lesion also extended to involve the dorsomedial nucleus of the thalamus. Because of this difference in morbid anatomy, and the inconsistency of the visual field asymmetries in the saccade latencies in the group as a whole, we cannot conclude from our results that the posterior thalamus has a critical role in generating either voluntary or visually guided saccades. The key finding of this study is that chronic lesions of this part of the thalamus disinhibit the neural system of ocular fixation and that there appear to be different neural substrates for controlling ocular fixation for voluntary and visually triggered saccades.

\section{Authors' affiliations \\ R Rafal, M McGrath, J Hindle, Centre for Cognitive Neuroscience, University of Wales, Bangor, UK; the North West Wales NHS Trust, UK L Machado, University of Otago, Dunedin, New Zealand}

This work was supported by the US National Institute of Mental Health and by the Wellcome Trust.

Competing interests: none declared

\section{REFERENCES}

1 Schlag J, Schlag-Rey M. Induction of oculomotor responses from thalamic internal medullary lamina in the cat. Exp Neurol 1971;33:498-508.

2 Maldonado HM, Schlag J. Unit activity related to head and eye movements in central thalamus of cats. Exp Neurol 1984;86:359-78.

3 Crommelinck M, Roucoux A, Meulders M. Eye movements evoked by stimulation of lateral posterior nucleus and pulvinar in th alert cat. Brain Res 1977; 124:361-6. 
4 Schlag J, Schlag-Rey M. Visuomotor functions of central thalamus in monkey II. Unit activity related to visual events, targeting, and fixation. J Neurophysiol 1984;51:1175-95.

5 Schlag-Rey M, Schlag J. Visuomotor functions of central thalamus in monkey. I. Unit activity related to spontaneous eye movements. J Neurophysiol 1984;51:1149-74.

6 Hirose G, Kosoegawa H, Saeki M, et al. The syndrome of posterior thalamic hemorrhage. Neurology 1985;35:998-1002.

7 Watson RT, Heilman KM. Thalamic neglect. Neurology 1979;29:690-4.

8 Ogren MP, Mateer CA, Wyler AR. Alterations in visually related eye movements following left pulvinar in man. Neuropsychologia 1984;22:187-9.

9 Brigell M, Babikian V, Goodwin JA. Hypometric saccades and low-gain pursuit resulting from a thalamic hemorrhage. Ann Neurol 1984;15:374-8.

10 Forbes K, Klein RM. The magnitude of the fixation offset effect with endogenously and exogenously controlled saccades. J Cogn Neurosci 1996;8:344-52

11 Machado L, Rafal R. Strategic control over the visual grasp reflex: studies of the fixation offset effect. Percept Psychophys 2000;62:1236-42.

12 Machado L, Rafal RD. Control of fixation and saccades in humans with chronic lesions of oculomotor cortex. Neuropsychology 2004;18:115-23.

13 Munoz DP, Wurtz RH. Fixation cells in monkey superior colliculus. I. Characteristics of cell discharge. J Neurophysiol 1993;70:559-75.
14 Munoz DP, Wurtz RH. Role of the rostral superior colliculus in active visual fixation and execution of express saccades. Vis Neurosci 1992;9:409-14.

15 Findlay JM, Walker R. A model of saccade generation based on parallel processing and competitive inhibition. Behav Brain Sci 1999;22:661-74, processing and compet
discussion $674-721$

16 Saslow MG. Effects of components of displacement-step stimuli upon latency for saccadic eye movements. J Opt Soc Am 1967:57:1024-9.

17 Klein R, Kingstone A. Why do visual offsets reduce saccadic latencies. Behav Brain Sci 1993; 16:583-4.

18 Munoz DP, Istvan PJ. Lateral inhibitory interactions in the intermediate layers of the monkey superior colliculus. J Neurophysiol 1998;79: 1193-209.

19 Dorris MC, Munoz DP. A neural correlate for the gap effect on saccadic reaction times in monkey. J Neurophysiol 1995;73:2558-62.

20 Bender DB, Baizer JS. Saccadic eye movements following kainic acid lesions of the pulvinar in monkeys. Exp Brain Res 1990;79:467-78.

21 Briand KA, Strallow D, Hening W, et al. Control of voluntary and reflexive saccades in Parkinson's disease. Exp Brain Res 1999;129:38-48.

22 Briand KA, Hening W, Poizner H, Sereno AB. Automatic orienting of visuospatial attention in Parkinson's disease. Neuropsychologia 2001;39:1240-9.

\section{NEUROLOGICAL PICTURE}

\section{Unilateral calf hypertrophy}

$\square$ his case illustrates an unusual cause of calf hypertrophy. A 47 year old man presented with a two year history of progressive swelling of the right calf. Initially painless, he later developed a dull ache over the lateral aspect of the lower leg. There was no weakness, nor any family history of muscle disease. On examination the right calf was enlarged, the right ankle reflex was absent and there was a Sl dermatomal sensory loss to pinprick. Investigations revealed a normal creatinine kinase; an enlarged soleus with altered signal on MRI of the calf; and features of active denervation on a calf muscle biopsy. MRI of the lumbosacral spine demonstrated the cause: a right lateral disc prolapse at L5/S1 causing a S1 radiculopathy. Nerve conduction studies were normal; electromyography of right gastrocnemius showed fibrillation potentials, positive sharp waves, and sparse isolated spontaneous muscle potentials but no neuromyotonia.

Calf hypertrophy in sciatica was noted as early as 1848 by Robert Graves ${ }^{1}$ and subsequently by Lhermitte ${ }^{2}$ in 1918. Since then neurogenic muscle hypertrophy has been reported in traumatic peripheral nerve lesions, polyneuropathies, spinal muscular atrophy, and SI radiculopathies. Suggested mechanisms have included a response to increased work in the remaining intact muscle fibres, increased stretch of muscle fibres by antagonist muscles, and neuromyotonia. ${ }^{3}$

A Coles

Department of Neurology, Addenbrooke's Hospital, Cambridge, UK D Dick

Department of Neurology, Norfolk and Norwich University Hospital, UK

Correspondence to: Dr A Coles, Department of Neurology, Box 165, Addenbrooke's Hospital, Hills Road, Cambridge CB2 2QQ, UK; ajc1020@medschl.cam.ac.uk

\section{References}

1 Graves R. Clinical lectures on the practice of medicine, 2nd edn. The New Sydenham Society, 1848;1:48.

2 Lhermitte J. Hypertrophie des muscles de la jambe consecutive a une lesion du nerf sciatique par balle. Rev Neurol 1918;25:56-8.

3 Rowin, Meriggioli. Complex repetitive discharges: cause or effect of neurogenic muscle hypertrophy. Muscle \& Nerve 1999;22:1603-6. doi: $10.1136 /$ jnnp.2004.035816

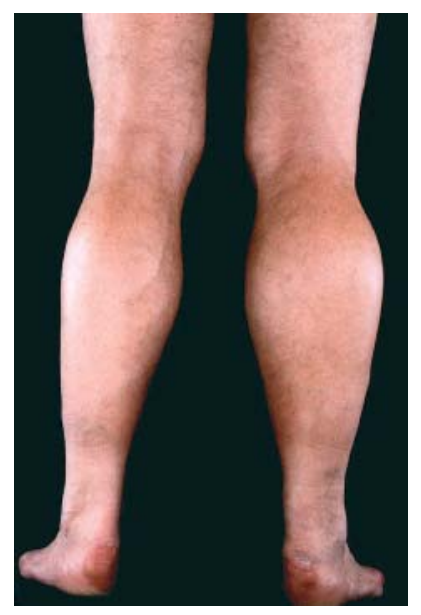

Figure 1 Enlarged right calf.

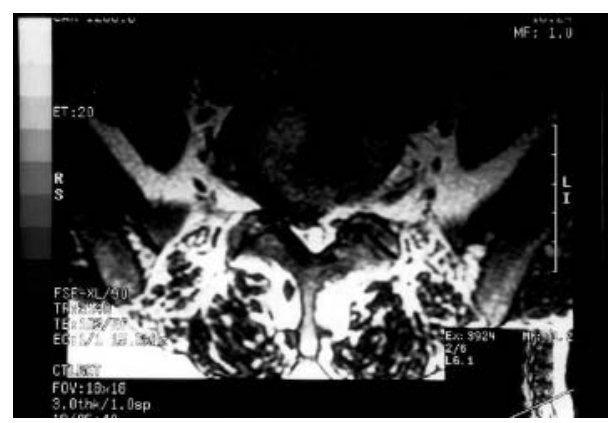

Figure 2 Axial MRI of L5/S1 disc. 